\title{
Spectral Line Distortions in the Presence of a Close-in Planet
}

\section{Citation}

Charbonneau, David, Saurabh Jha, and Robert W. Noyes. 1998. “Spectral Line Distortions in the Presence of a Close-in Planet." The Astrophysical Journal 507 (2): L153-56. https:// doi.org/10.1086/311703.

\section{Permanent link}

http://nrs.harvard.edu/urn-3:HUL.InstRepos:41397431

\section{Terms of Use}

This article was downloaded from Harvard University's DASH repository, and is made available under the terms and conditions applicable to Other Posted Material, as set forth at http:// nrs.harvard.edu/urn-3:HUL.InstRepos:dash.current.terms-of-use\#LAA

\section{Share Your Story}

The Harvard community has made this article openly available.

Please share how this access benefits you. Submit a story.

Accessibility 


\title{
SPECTRAL LINE DISTORTIONS IN THE PRESENCE OF A CLOSE-IN PLANET
}

\author{
David Charbonneau, Saurabh Jha, and Robert W. Noyes \\ Harvard-Smithsonian Center for Astrophysics, 60 Garden Street, Cambridge, MA 02138; \\ dcharbonneau@cfa.harvard.edu, sjha@cfa.harvard.edu,rnoyes@cfa.harvard.edu \\ Received 1998 August 14; accepted 1998 September 8; published 1998 September 23
}

\begin{abstract}
We discuss the interpretation of the distortions to the stellar spectral lines, with particular attention to line bisectors in the presence of an orbiting planetary companion. We present a simple model whereby light reflected by the companion can cause temporal variations to the observed line profiles. These distortions have a characteristic signature that depends on the inclination angle of the system. For the known close-in extrasolar giant planets, the expected amplitude of the effect might not be far from current detection capabilities. This method could be used to detect the presence of the companion directly, yielding the orbital inclination and hence the planetary mass. Futhermore, a detection would measure a combination of the planetary radius and albedo, from which a minimum radius may be deduced.
\end{abstract}

Subject headings: planetary systems — stars: individual ( $\tau$ Bootis, 51 Pegasi $)$ techniques: spectroscopic

\section{INTRODUCTION}

The interpretation of low-amplitude, periodic radial velocity variations in solar-type stars as being induced by orbiting lowmass companions was recently put into question. Gray (1997) and Gray \& Hatzes (1997) claimed to detect distortions to the line profile bisector (the locus of midpoints of a stellar absorption line from the core up to the continuum) in one spectral line ( $\mathrm{Fe}$ I $\lambda 6253)$ of the star 51 Pegasi at the now well-known 4.23 day radial velocity period (Mayor \& Queloz 1995; Marcy et al. 1997). They stated that the planet hypothesis could not account for variations in the line profiles and proposed the alternative explanation that $51 \mathrm{Peg}$ was undergoing nonradial pulsations. Further observations and analyses (Gray 1998; Hatzes, Cochran, \& Bakker 1998a, 1998b; Brown et al. 1998a, 1998b) failed to confirm the claimed period in the line bisector, and the planet hypothesis has emerged as the most reasonable explanation of the radial velocity curve.

Nevertheless, the question of the interpretation of variations in the line bisector remains. Is it a given that the planet explanation is immediately excluded should intrinsic variations in the line profiles of $51 \mathrm{Peg}$ be detected at the claimed orbital period? Our purpose in this Letter is twofold: (1) to provide a simple model whereby a close-in orbiting companion produces periodic distortions to the line profiles and (2) to explore the observational consequences of this effect, the amplitude of which might be close to current observational limits, and the specific signature of which is set by the orbital inclination of the system and the radius and albedo of the companion.

\section{REFLECTED LIGHT}

The amount of light reflected by the planets of our solar system is a tiny fraction of the solar output. However, for the close-in extrasolar giant planets (CEGPs) discovered to date, this fraction increases substantially because of the proximity of a large planet to the star. The semimajor axes of these systems, $\tau$ Boo, $51 \mathrm{Peg}, v$ And, and $\rho^{1}$ Cnc, are 0.046, 0.051, 0.057, and 0.11 AU, respectively (Mayor \& Queloz 1995; Butler et al. 1997). An estimate of the flux ratio can be achieved as follows. Let $D$ denote the distance from the star to the planet, $R_{p}$ the radius of the planet, $R_{s}$ the radius of the star, and $\alpha$ the angle between the star and the Earth as seen from the planet.
We assume that $R_{p} \ll R_{s} \ll D$ and that each surface element on the planet reflects a fraction $\eta$ of the incident flux uniformly back into the local half-sky (Lambert's law). If $\eta$ does not vary with position, then it is simply the Bond albedo. Integration over the sphere yields the ratio of the observed flux of the planet at $\alpha=0$ to that of the star,

$$
\epsilon=\frac{2 \eta}{3}\left(\frac{R_{p}}{D}\right)^{2} .
$$

For either 51 Peg b $(D=0.051 \quad \mathrm{AU}$ and assuming $R_{p} \simeq 1.3 R_{\mathrm{Ju}}$; Guillot et al. 1996) or $\tau$ Boo b $(D=0.046 \mathrm{AU}$ and assuming $R_{p} \simeq 1.2 R_{\mathrm{Jup}}$ ), the result is $\epsilon \simeq \eta \times 10^{-4}$.

The observed flux ratio will vary with the angle $\alpha$, which is a combination of the orbital inclination $i$ and the orbital phase $\phi$. Here we define the orbital phase as the one conventionally given by radial velocity measurements; for a circular orbit (the only case we consider, as tidal effects circularize the orbits of these extremely close companions), $\phi=0$ is taken to be the time of the maximum recessional velocity of the star. The angle $\alpha \in[0, \pi]$ is then given by $\cos \alpha=-\sin$ $i \sin 2 \pi \phi$. Integration of the intensity over the surface of the sphere viewed at an angle $\alpha$ yields the phase-dependent observed flux ratio,

$$
f(\phi, i)=\epsilon\left[\frac{\sin \alpha+(\pi-\alpha) \cos \alpha}{\pi}\right] .
$$

For a discussion of the albedos and phase functions of solar system objects, see Harris (1961). Note that in the expression for $f(\phi, i)$, we have ignored the possibility of occultation; eclipses would have a much more pronounced effect and are ruled out for $\tau$ Boo, 51 Peg, and $\rho^{1}$ Cnc (Henry et al. 1997; Baliunas et al. 1997).

Thus, CEGPs at moderate to high inclination and of high reflectivity will produce a photometric modulation of the system that will be accessible to upcoming satellite missions, as discussed by Charbonneau (1998). However, photometry does not make use of the spectroscopic separation of the planet and star. The key to the method we propose here is in analyzing the spectrum of the system. Assuming the planet reflects an essentially undistorted spectrum of the star, a copy exists in 

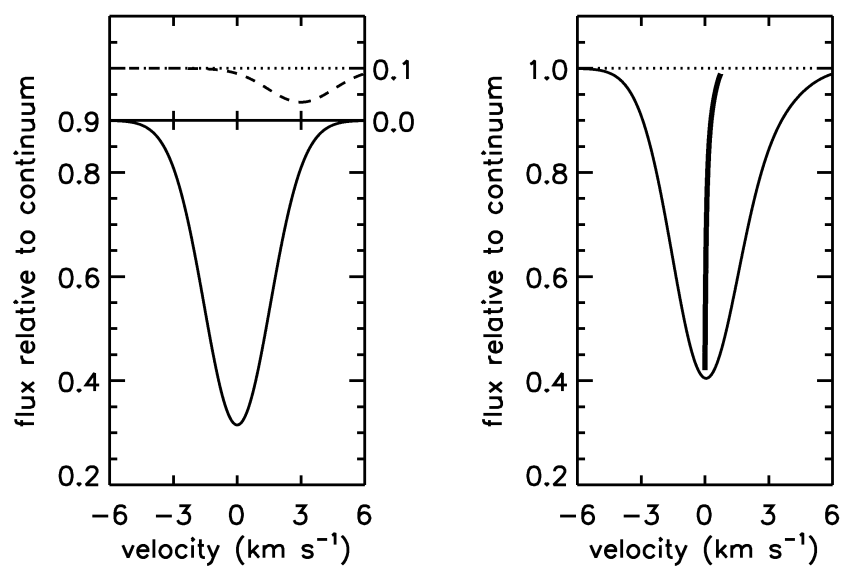

FIG. 1.-The observed line profile is the sum of the stellar absorption feature and a time-varying, Doppler-shifted-reflected light component. The amplitude of the reflected light has been greatly exaggerated in this plot so as to make the resulting distortion to the bisector visible.

the observed stellar spectrum with relative amplitude $f(\phi, i)$ but at a location that is Doppler-shifted because of the motion of the planet.

The ratio of the amplitude of the radial velocity variations of the planet to that of the star, $K_{p} / K_{s}$, is simply the ratio of the mass of the star to the mass of the planet, $M_{s} / M_{p}$. From the single-line spectroscopic orbit, only the minimum mass of the planet, $M_{p} \sin i$, is determined (assuming the stellar mass is estimated via other means). Thus, only the combination $K_{p} / \sin i$ is known. For $51 \mathrm{Peg}, K_{s} \simeq 56 \mathrm{~m} \mathrm{~s}^{-1}$ and $M_{p} \sin i \simeq 4.4 \times 10^{-4} M_{s}$, so $K_{p} / \sin i \simeq 130 \mathrm{~km} \mathrm{~s}^{-1}$. Thus, there can be a huge difference in velocity amplitude between the stellar and reflected light signal.

For a given stellar spectral line, the reflected line will move back and forth as the phase changes. At $\phi=0.25$ and $\phi=$ 0.75 , the radial velocities of the star and planet will be the same, and the reflected line will lie on top of the stellar line. At $\phi=0$ and $\phi=0.5$, the lines will be maximally separated. A single spectrum with a sufficient signal-to-noise ratio would, in principle, be able to show both. We have obtained many high signal-to-noise ratio, high-resolution spectra of $\tau$ Boo, and we are attempting to isolate the Doppler-shifted-reflected spectrum from the orders-of-magnitude brighter stellar spectrum by making use of the known orbital parameters supplied by radial velocity measurements (Butler et al. 1997). The results of this complementary investigation will be presented in a separate paper. Here we present an alternate method of detection that capitalizes on the high-resolution observations and analysis methods that have already been developed for studying line bisectors. The motion of the reflected line through the stellar line will cause a small distortion that might be detectable by careful measurements of the line bisectors or through other techniques.

\section{MODEL AND RESULTS}

We assume that the planet reflects an undistorted spectrum of the primary, leading to a composite spectrum that consists of a reflected component greatly reduced in amplitude and Doppler-shifted by the orbital motion of the companion. We model this process for an individual isolated spectral line. Typical line bisectors for solar-type stars show a distinctive $\mathrm{C}$ shape caused by granulation in the photosphere (see, e.g., Gray 1992).
Here we have described the lines as simple Gaussian profiles of appropriate width and depth for the star in question. While we have calculated more detailed models (multiple Gaussian components to the line) so as to reproduce the constant bisector C shape, the effect of this is simply to add a time-invariant term to the bisector shape derived from the symmetric Gaussian model.

The velocity of the planet relative to the star at phase $\phi$ is simply

$$
v_{p}(\phi, i)=-K_{s} \frac{M_{s}+M_{p}}{M_{p}} \cos 2 \pi \phi .
$$

We have modeled the stellar and planetary line profiles as a function of velocity by

$$
\begin{aligned}
s(v) & =1-a_{s} \exp \left(-v^{2} / \Delta v_{s}^{2}\right), \\
p(v, \phi, i) & =1-a_{p} \exp \left\{-\left[v-v_{p}(\phi, i)\right]^{2} / \Delta v_{p}^{2}\right\}
\end{aligned}
$$

where we have explicitly allowed for a different planetary line width $\left(\Delta v_{p}\right)$ and depth $\left(a_{p}\right)$ because of the rotation rates of the planet and star (see below).

The flux ratio is given by equation (2); thus, the observed spectrum of the system is

$$
I(v, \phi, i)=\frac{s(v)+f(\phi, i) p(v, \phi, i)}{1+f(\phi, i)}
$$

where we have renormalized to the continuum flux level. The effect of the reflected light component on the observed bisector is shown schematically in Figure 1.

For each point in the phase, we deduce the bisector by evaluating the midpoint between the two halves of the absorption feature at a number of flux levels, interpolating between sampled points to the desired flux. We have also characterized these distortions in terms of the two parameters that are conventionally used in bisector analyses, the velocity span and the bisector curvature. The velocity span measures the velocity difference in the bisector at two flux levels; we have chosen these levels at $2 \%$ below the continuum $(I=0.98)$ and $2 \%$ above the line depth $\left(I=1.02-a_{s}\right)$. The bisector curvature is defined as the velocity span of the top portion [between $I=0.98$ and $I=$ $1-\left(a_{s} / 2\right)$ ] minus the velocity span of the bottom portion [between $I=1-\left(a_{s} / 2\right)$ and $\left.I=1.02-a_{s}\right]$ of the bisector.

For convenience, we assume $\eta=1$ in our model calculations. The amplitude of the distortion to the bisector (and hence to the velocity span and curvature) is linear in $\eta$. The results we present should therefore be scaled for a given choice of the reflectivity.

We have computed simulations for 51 Peg with $\epsilon=10^{-4}$ and a stellar line width of $\Delta v_{s}=2.2 \mathrm{~km} \mathrm{~s}^{-1}$. We assume that the reflected light is undistorted, so that $\Delta v_{p}=\Delta v_{s}$ and $a_{p}=$ $a_{s}$. We sample the data simulating a spectral resolution $\lambda / \Delta \lambda$ $=200,000$ comparable to current high-resolution observations. The predicted distortions to the bisector for $i=80^{\circ}$ are shown in the left panel of Figure 2 for a selection of radial velocity phases. The deduced velocity span and bisector curvature are shown in the top panels of Figures 3 and 4, respectively. The bisector is distorted for the fraction of the phase where the stellar and planetary lines overlap and the planet is sufficiently illuminated as viewed by the observer. For a near edge-on geometry in the case of $51 \mathrm{Peg}$, this corresponds to roughly 

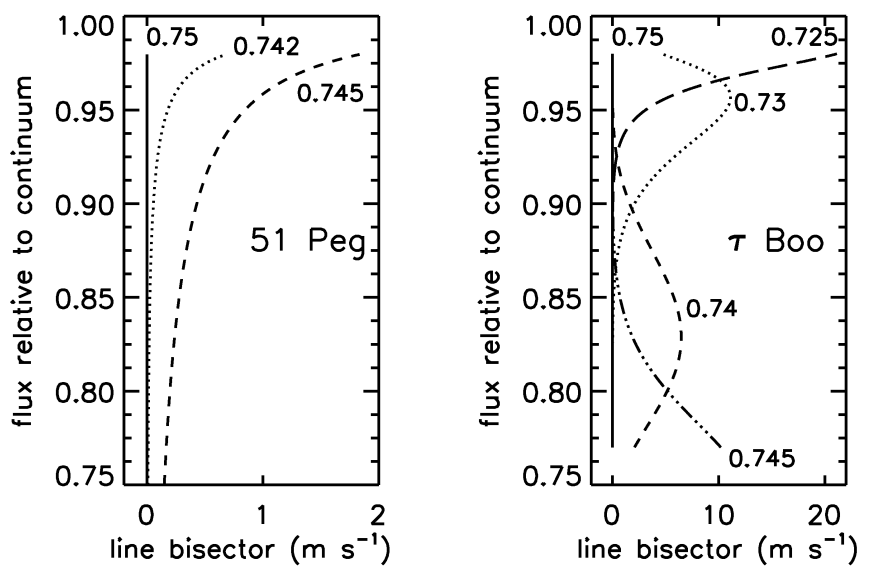

FIG. 2.-Predicted distortions to the bisector of a typical spectral line of 51 Peg and $\tau$ Boo for $i=80^{\circ}$ and $\epsilon=10^{-4}$. The bisectors are labeled by radial velocity phase.

$3 \%$ of its 4.23 day period, or about $3 \mathrm{hr}$, centered on $\phi=$ 0.75 . Although the stellar and planetary lines also overlap at $\phi=0.25$, there is less distortion since the planet is less illuminated as viewed by the observer. For lower inclinations, the fraction of the period in which there is a significant distortion is greater, but the amplitude of the distortion is less.

We have also modeled this effect for another CEGP system, $\tau$ Boo $\left(K_{s}=469 \mathrm{~m} \mathrm{~s}^{-1}\right.$ and $M_{p} \sin i / M_{s}=3.1 \times 10^{-3}$; Butler et al. 1997). Observations of $\mathrm{Ca}$ II $\mathrm{H}$ and $\mathrm{K}$ lines suggest that the rotational period of the star is the same as the observed orbital period of the companion (Baliunas et al. 1997), implying that the star and planet are tidally locked. The synchronization timescales for $\tau$ Boo are discussed in Marcy et al. (1997). If indeed the system is tidally locked, the planet does not see the rotational broadening of the spectral lines of the primary, since there is no relative motion between any point on the surface of the planet and any point on the surface of the star. Thus, the planet reflects a rotationally unbroadened spectrum of the primary, with an intrinsic width that is due to the stellar photospheric convective motions. An observer then sees this line broadened only by the planetary rotation, which, in this tidally locked scenario, is small. Thus, in the case of $\tau$ Boo, we predict relatively narrow planetary lines (we adopt $\Delta v_{p} \simeq 4 \mathrm{~km} \mathrm{~s}^{-1}$ ) superposed on the much broader stellar lines $\left(\Delta v_{s} \simeq 15 \mathrm{~km}\right.$ $\left.\mathrm{s}^{-1}\right)$. The results of our simulated bisector distortions for $i=$ $80^{\circ}$ are shown in the right panel of Figure 2 for a selection of radial velocity phases. The deduced velocity span and curvature with phase are shown in the bottom panels of Figures 3 and 4 , respectively. The line is significantly distorted for the fraction of the phase where the planetary and stellar lines overlap, which now is substantially increased because of the high degree of rotational broadening experienced by the lines of the star. For an edge-on configuration, this amounts to roughly $10 \%$, or about $8 \mathrm{hr}$ of the 3.3 day period, centered on $\phi=0.75$. Due to the different line widths, the deduced bisector varies with a much greater amplitude and in a more complex manner than in the case of $51 \mathrm{Peg}$. The velocity span and curvature do not characterize the behavior of the bisector very well. We advocate that the full bisectors be examined in a search for this effect.

\section{DISCUSSION}

We have shown that the presence of a close-in extrasolar giant planet can distort the observed spectral line profiles of
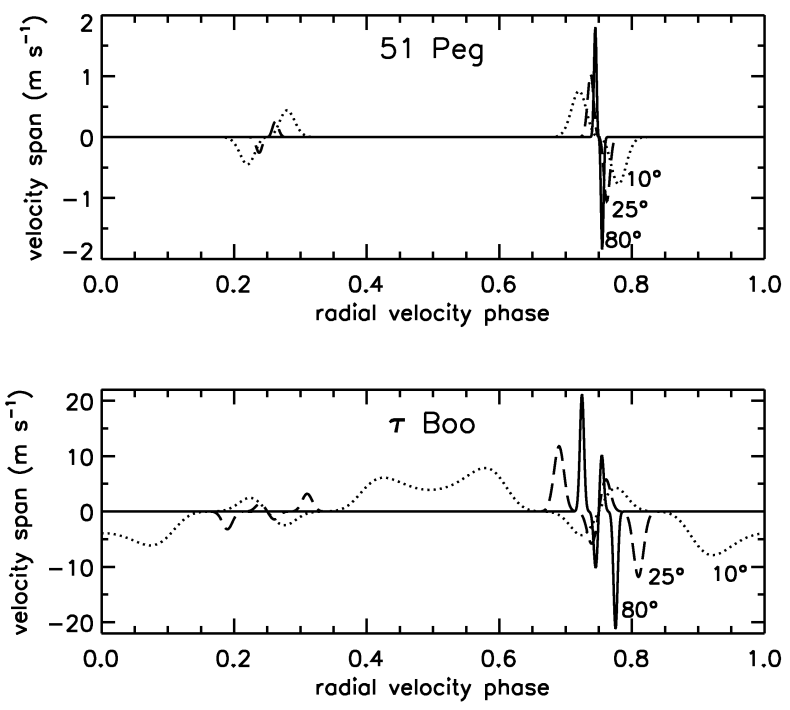

FIG. 3.-The velocity span variations with radial velocity phase for $51 \mathrm{Peg}$ and $\tau$ Boo, both with $\epsilon=10^{-4}$. The inclinations are $80^{\circ}$ (solid), $25^{\circ}$ (dashed), and $10^{\circ}$ (dotted).

the system through the contribution of reflected light from the companion. For $51 \mathrm{Peg}$, assuming $i=80^{\circ}$ and $\epsilon=\eta \times 10^{-4}$, the amplitude of the distortion is roughly $4 \eta$ and $3 \eta \mathrm{m} \mathrm{s}^{-1}$ (peak to peak) to the velocity span and curvature, respectively. If $\eta$ is high, the effect is commensurate with current observational limits: Hatzes et al. (1998a) combined measurements of five spectral lines of $51 \mathrm{Peg}$ and achieved a scatter of 7 and $12 \mathrm{~m}$ $\mathrm{s}^{-1}$ for the velocity span and curvature, respectively. More recent results (Hatzes et al. 1998b) achieve a precision of $1 \mathrm{~m}$ $\mathrm{s}^{-1}$ for the velocity span and $4 \mathrm{~m} \mathrm{~s}^{-1}$ for the curvature. In the case of $\tau$ Boo, assuming that the system is tidally locked and that $i=80^{\circ}$ and $\epsilon=\eta \times 10^{-4}$, the velocity span and curvature distortions are both about $42 \eta \mathrm{m} \mathrm{s}^{-1}$ peak to peak. Again, for a high value of $\eta$, these are close to the current upper limits as measured by Hatzes \& Cochran (1998).

The amplitude of the velocity span and curvature variations
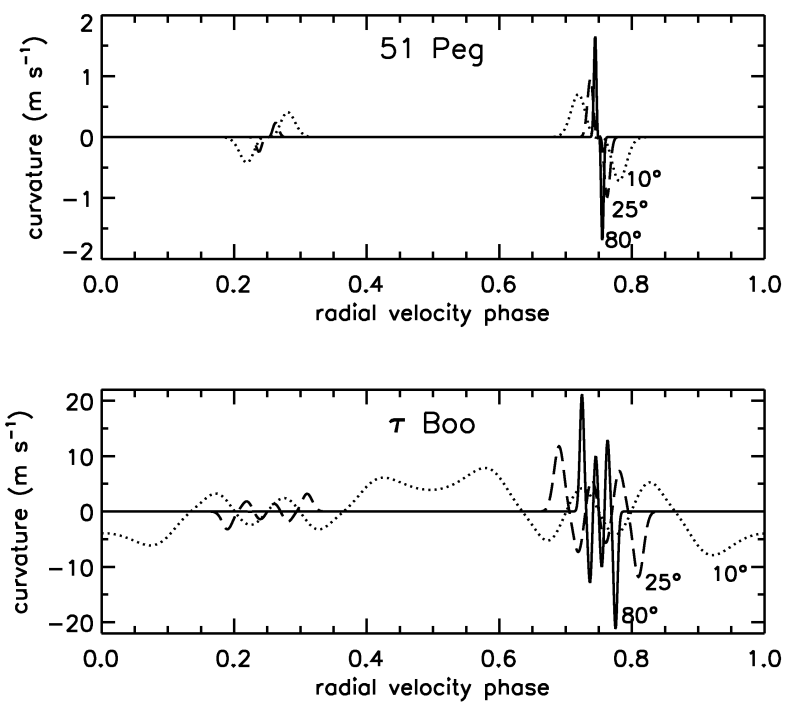

FIG. 4.-The curvature variations with radial velocity phase for $51 \mathrm{Peg}$ and $\tau$ Boo, both with $\epsilon=10^{-4}$. The inclinations are $80^{\circ}$ (solid), $25^{\circ}$ (dashed), and $10^{\circ}$ (dotted). 
we have stated here rely on the measurement of the line bisector to within $2 \%$ of the continuum flux level. Bisector measurements tend to avoid the extreme wings of the line profile since $d I / d \lambda$ is small, and hence the deduced bisector is more easily corrupted by noise. For $51 \mathrm{Peg}$, the equal line widths of the stellar and reflected spectrum mean that much of the signal in the velocity span and curvature is a result of the distortion of the bisector close to the continuum, as shown in Figure 2. For $\tau$ Boo, the different line widths in the tidally locked scenario produce a second trough and peak in the velocity span and curvature that are insensitive to the height to which the bisector is measured, because the distortion occurs away from the continuum. Note that confirmation of the predicted distortions does not require an accurate measurement of the intrinsic line bisector, only a precise measurement of the temporal variations to it, and thus it is not threatened by contamination from line blends. Also, isolated lines are not required since distortions induced by the transit of nearby lines can easily be modeled under the assumption that the planet reflects a Doppler-shifted copy of the stellar spectrum.

The estimate of the reflected light amplitude presented in equation (1) does not consider the underlying source of the albedo, nor any angle-dependent scattering effects associated with it. Estimates of the albedos of these CEGPs are uncertain since the chemistry of these atmospheres is unknown. The gas giants of the solar system have Bond albedos ranging from 0.7 to 0.9 . The large reflectivity is primarily due to the presence of ices and clouds high in the atmosphere, and these could not exist at the much greater effective temperatures of CEGPs. The dominant sources of reflectivity for CEGPs are the scattering from dust and Rayleigh scattering. Detailed modeling of the atmospheric chemistry of these planets indicates that condensates such as $\mathrm{MgSiO}_{3}$ may indeed form at the required height (Burrows et al. 1998). Recent models by Seager \& Sasselov (1998), which explicitly solve the radiative transfer through a model atmosphere for $\tau$ Boo b, predict an albedo of less than
$1 \%$ at $5000 \AA$. Although this model is incomplete in that it considers only one dust species of interstellar size distribution and solar abundance, it seems unlikely that albedos as high as those of the solar system gas giants could be reached by a dusty CEGP; further investigation is called for. An upper limit on the bisector distortions that is sufficiently strict to disallow a large value of $\eta$ would provide support for such models.

\section{CONCLUSION}

Via reflected light, a close-in extrasolar giant planet could produce a distortion to the stellar spectral line bisectors at a level that is approaching the current observational upper limits, given a sufficiently high albedo. The variation of the bisector distortions with phase has a very distinctive signature that depends on the inclination of the orbit of the planet to the line of sight. The measurement of such periodic bisector variations would constitute a direct detection of the companion, and this would be a major advance in the field of extrasolar planet research. This would yield the inclination and hence the mass of the companion. It would also measure the product of the albedo and the square of the radius of the planet, from which a minimum planetary radius may be deduced. An estimated albedo would yield a value for the planetary radius, which can be combined with the measured mass to calculate such critically important quantities as the surface gravity and average density. Conversely, a firm upper limit on such distortions would place a constraint on the albedos of these planets. Either by detection or by an upper limit, an accurate measurement of the line profile bisector would place an important observational constraint on the models of these close-in extrasolar giant planets.

We wish to thank David Latham, in whose informative seminar this idea was conceived, and Timothy Brown, Artie Hatzes, and Dimitar Sasselov for pragmatic comments. This work was supported in part by an NSF Graduate Research Fellowship.

\section{REFERENCES}

Baliunas, S. A., Henry, G. W., Donahue, R. A., Fekel, F. C., \& Soon, W. H. 1997, ApJ, 474, L119

Brown, T. M., Kotak, R., Horner, S. D., Kennelly, E. J., Korzennik, S., Nisenson, P., \& Noyes, R. W. 1998a, ApJ, 494, L85

- 1998b, ApJS, 117, 563

Burrows, A., et al. 1998, in ASP Conf. Ser. 154, Cool Stars, Stellar Systems, and the Sun, 10th Cambridge Workshop, ed. R. A. Donahue \& J. A. Bookbinder (San Francisco: ASP), 27

Butler, R. P., Marcy, G. W., Williams, E., Hauser, H., \& Shirts, P. 1997, ApJ, 474, L115

Charbonneau, D. 1998, in NATO/ASI Ser., Planets Outside the Solar System: Theory and Observations, ed. J.-M. Mariotti (Dordrecht: Kluwer), in press

Gray, D. F. 1992, The Observations and Analysis of Stellar Photospheres (2d ed.; Cambridge: Cambridge Univ. Press) 1997, Nature, 385, 795
Gray, D. F. 1998, Nature, 391, 153

Gray, D. F., \& Hatzes, A. P. 1997, ApJ, 490, 412

Guillot, T., Burrows, A., Hubbard, W. B., Lunine, J. I., \& Saumon, D. 1996, ApJ, 459, L35

Harris, D. L. 1961, in The Solar System, Vol. 3, Planets and Satellites, ed. G. P. Kuiper \& B. M. Middlehurst (Chicago: Univ. Chicago Press), 272 Hatzes, A. P., \& Cochran, W. D. 1998, ApJ, 502, 944

Hatzes, A. P., Cochran, W. D., \& Bakker, E. J. 1998a, Nature, 391, 154 1998b, ApJ, in press

Henry, G. W., Baliunas, S. L., Donahue, R. A., Soon, W. H., \& Saar, S. H. 1997, ApJ, 474, 503

Marcy, G. W., Butler, R. P., Williams, E., Bildsten, L., Graham, J. R., Ghez, A. M., \& Jernigan, J. G. 1997, ApJ, 481, 926

Mayor, M., \& Queloz, D. 1995, Nature, 378, 355

Seager, S., \& Sasselov, D. 1998, ApJ, 502, L157 